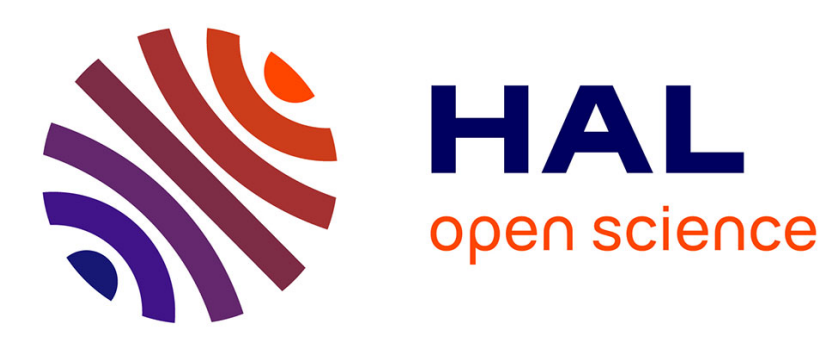

\title{
Contribution of Surface Distributions to Constant-Phase-Element (CPE) Behavior: 3. Adsorbed Intermediates
}

Christopher L. Alexander, Bernard Tribollet, Vincent Vivier, Mark E. Orazem

\section{To cite this version:}

Christopher L. Alexander, Bernard Tribollet, Vincent Vivier, Mark E. Orazem. Contribution of Surface Distributions to Constant-Phase-Element (CPE) Behavior: 3. Adsorbed Intermediates. Electrochimica Acta, 2017, 251, pp.99-108. 10.1016/j.electacta.2017.08.081 . hal-01617294

\section{HAL Id: hal-01617294 \\ https: / hal.sorbonne-universite.fr/hal-01617294}

Submitted on 17 Oct 2017

HAL is a multi-disciplinary open access archive for the deposit and dissemination of scientific research documents, whether they are published or not. The documents may come from teaching and research institutions in France or abroad, or from public or private research centers.
L'archive ouverte pluridisciplinaire HAL, est destinée au dépôt et à la diffusion de documents scientifiques de niveau recherche, publiés ou non, émanant des établissements d'enseignement et de recherche français ou étrangers, des laboratoires publics ou privés. 


\title{
Contribution of Surface Distributions to Constant-Phase-Element (CPE) Behavior: 3. Adsorbed Intermediates
}

Christopher L. Alexander ${ }^{\mathrm{a}}$, Bernard Tribollet ${ }^{\mathrm{b}}$, Vincent Vivier ${ }^{\mathrm{b}}$, Mark E.

Orazem $^{\mathrm{a}, *}$

${ }^{a}$ Department of Chemical Engineering, University of Florida, Gainesville, FL, 32611, USA

${ }^{b}$ LISE, UPR 15 du CNRS, Université P. et M. Curie, CP 133, 4 Place Jussieu, 75252

Paris cedex 05 France

\begin{abstract}
The influence of surface distributions on rates of heterogeneous reactions coupled by adsorbed intermediates was studied to determine whether this form of surface heterogeneity can provide a physical explanation for constantphase-element behavior. Results obtained from finite-element simulations on disk and recessed disk electrodes show that there are two components that give rise to frequency dispersion. Frequency dispersion occurs due to geometry-induced nonuniform current distributions which leads to a complex ohmic impedance. The effects of geometry-induced frequency dispersion may be mitigated by use of small electrodes. Frequency dispersion also occurs due to the potential dependence of the faradaic impedance. The characteristic frequency associated with this form of frequency dispersion is not dependent on disk radius, but the contribution of frequency dispersion associated with reactions coupled by adsorbed intermediates may be reduced with the use of small electrodes.
\end{abstract}

Keywords: Frequency dispersion, surface heterogeneity, impedance spectroscopy, finite-element simulations

\footnotetext{
*Corresponding author

Email address: meo@che.ufl.edu (Mark E. Orazem)

published as Electrochimica Acta, 251 (2017), 99-108.
} 


\section{Introduction}

Frequency dispersion is almost always observed in impedance measurements over a broad range of frequencies and is assumed to be caused by a distribution of time constants. Constant-phase elements (CPE) are often used to fit impedance measurements exhibiting frequency dispersion, but the extracted parameters do not necessarily have a clear physical significance. Therefore, there is a need to form an understanding of the factors that contribute to frequency dispersion in impedance measurements and to uncover the physical origin of the constant-phase element.

Jorcin et al.[1] used local impedance spectroscopy to show that frequency dispersion can arise from surface or normal distributions of time constants. The frequency dispersion associated with a distribution of time constants normal to the electrode surface is well established; whereas, the frequency dispersion associated with a surface distribution is not well understood. Hirschorn et al. $[2,3]$ showed that a power-law distribution of resistivity through a film yields CPE behavior. The power-law-model approach has been used successfully to extract a film capacitance and associated parameters for a variety of systems, including oxides on steel,[4] human skin,[4, 5] and polymer coatings. $[6,7]$

Brug et al. [8] developed an expression for the capacitance extracted from a CPE caused by a surface distribution of capacitance. Córdoba-Torres et al. [9] showed that the Brug model accounted for the correlation observed between CPE parameters $\alpha$ and $Q$ for two experimental conditions: the corrosion of polycrystalline iron and the deposition of $\mathrm{CaCO}_{3}$ scale on gold electrodes. The results were attributed to a distribution of time constants associated with surface heterogeneity. The exact nature of the surface heterogeneity was not identified. In subsequent work, Córdoba-Torres et al. [10] suggested that the CPE behavior results from energetic distributions rather than geometric heterogeneity or roughness.

Alexander et al. [11] showed that a capacitance distribution gave rise to frequency dispersion, but the effect was seen at frequencies higher than that associated with the disk geometry. The characteristic length for a periodic radial distribution was the period of the distribution and, as the period decreased, the frequency dispersion occurred at higher frequencies. A surface distribution of reactivity for a single-step reaction mechanism did not produce frequency dispersion. [12] However, Wu et al. [13] showed that the geometry of a disk electrode embedded within an insulating plane with re- 
actions involving adsorbed intermediates causes frequency dispersion at low frequencies. Their conclusions were supported by comparison to experimental results obtained for an iron disk in a $0.5 \mathrm{M} \mathrm{H}_{2} \mathrm{SO}_{4}$ solution.[14]

In many cases, for example the work of Córdoba-Torres et al. [9], the model presented by Brug et al.,[8] based on a distribution of properties along the electrode surface, provides a convincing correlation of CPE parameters extracted from impedance data. The present work is part of an effort to understand what distribution of surface properties may give rise to CPE behavior in the experimentally observed frequency range. For disk electrodes, the frequency dispersion may be attributed to the influence of nonuniform current and potential distributions. For recessed electrodes, frequency dispersion may be caused by a surface distribution of reaction rates for reactions coupled by an adsorbed intermediate. Finite-element models were used to simulate the impedance of disk and recessed disk electrodes with and without surface heterogeneity of reactions coupled by an adsorbed intermediate.

\section{Mathematical Development}

The system under consideration involves two reactions coupled by an adsorbed ion. In the first step,

$$
\mathrm{M} \rightarrow \mathrm{X}_{\mathrm{ads}}^{+}+e^{-}
$$

where the metal reacts to form an adsorbed ion on the surface and an electron is released. In the second step,

$$
\mathrm{X}_{\mathrm{ads}}^{+} \rightarrow \mathrm{P}^{2+}+e^{-}
$$

where the adsorbed ion desorbs and another electron is released. The total faradaic current may be expressed as the sum of the currents associated with reactions (1) and (2)

$$
i_{\mathrm{F}}=i_{\mathrm{M}}+i_{\mathrm{X}}
$$

Armstrong et al. [15] described similar general mechanisms for the impedance response associated with coupled electrochemical reactions and discussed its application to iron dissolution in carbonate/bicarbonate buffers. Mechanisms similar to reactions (1) and (2) were proposed by Epelboin and Keddam[16] for the impedance of iron dissolution through two steps involving an adsorbed FeOH intermediate. They simulated the impedance response for different applied potentials, showing how the same mechanism can account for different 
shapes of impedance diagrams. Similar approaches were developed by Péter et al. [17] for the impedance model of the dissolution of aluminum in three consecutive steps with two adsorbed intermediates and by Roy et al. [18] and Mathias et al. [19] for the influence of reversible oxide formation on a Pt catalyst in PEM fuel cells.

Under the assumption of Tafel kinetics, the current associated with the first step may be expressed as

$$
i_{\mathrm{M}}=K_{\mathrm{M}}(1-\gamma) \exp \left(b_{\mathrm{M}} V\right)
$$

and is dependent on the available surface that is not covered by the adsorbed species. A similar expression may be expressed for the second step as

$$
i_{\mathrm{X}}=K_{\mathrm{X}} \gamma \exp \left(b_{\mathrm{X}} V\right)
$$

where $K_{\mathrm{M}}$ and $K_{\mathrm{X}}$ are the effective rate constants with units of current density, $\gamma$ is the fractional surface coverage of the adsorbed intermediate, $b_{\mathrm{M}}$ and $b_{\mathrm{X}}$ are constants which can be expressed in terms of the charge-transfer coefficients, $\alpha_{\mathrm{M}}$ and $\alpha_{\mathrm{X}}$, as

$$
b=\frac{\alpha \mathrm{F}}{\mathrm{RT}} .
$$

The potential, $V$ represents the potential difference between the metal and the region outside the double layer, $V=\Phi_{\mathrm{m}}-\Phi_{0}$.

All variables may be expressed as a sum of a steady-state and timedependent components as

$$
X=\bar{X}+\operatorname{Re}\{\widetilde{X} \exp (\mathrm{j} \omega t)\}
$$

in which $\tilde{X}$ is a complex variable dependent on frequency. Therefore, the faradaic current may be expressed as

$$
i_{\mathrm{F}}=\bar{i}_{\mathrm{F}}+\operatorname{Re}\left\{\widetilde{i}_{\mathrm{F}} \exp (\mathrm{j} \omega t)\right\}
$$

in which $\tilde{i_{\mathrm{F}}}$ is the oscillating component of the faradaic current. The total steady-state faradaic current may be expressed as

$$
\bar{i}_{\mathrm{F}}=K_{\mathrm{M}}(1-\bar{\gamma}) \exp \left(b_{\mathrm{M}} \bar{V}\right)+K_{\mathrm{X}} \bar{\gamma} \exp \left(b_{\mathrm{X}} \bar{V}\right)
$$

and is dependent on the steady-state surface coverage $\bar{\gamma}$, which may be derived from the rate of change in surface coverage over time expressed as

$$
\frac{\partial \gamma}{\partial t}=\frac{i_{\mathrm{M}}-i_{\mathrm{X}}}{\Gamma \mathrm{F}}
$$


where $\Gamma$ represents the maximum surface coverage. By setting equation (10) equal to zero, the steady-state surface coverage may be obtained as

$$
\bar{\gamma}=\frac{K_{\mathrm{M}} \exp \left(b_{\mathrm{M}} \bar{V}\right)}{K_{\mathrm{M}} \exp \left(b_{\mathrm{M}} \bar{V}\right)+K_{\mathrm{X}} \exp \left(b_{\mathrm{X}} \bar{V}\right)}
$$

The charge-transfer resistance associated with each reaction step is inversely related to the steady state current as

$$
R_{\mathrm{t}, \mathrm{M}}=\frac{1}{b_{\mathrm{M}} \bar{i}_{\mathrm{M}}}
$$

and

$$
R_{\mathrm{t}, \mathrm{X}}=\frac{1}{b_{\mathrm{X}} \bar{i}_{\mathrm{X}}}
$$

respectively. The total charge-transfer resistance is the parallel combination of the charge-transfer resistances of each reaction expressed as

$$
\frac{1}{R_{\mathrm{t}}}=\frac{1}{R_{\mathrm{t}, \mathrm{M}}}+\frac{1}{R_{\mathrm{t}, \mathrm{X}}}
$$

As described by Orazem and Tribollet,[20, 21] the oscillating faradaic current density is derived by differentiating the steady current with respect to potential and surface concentration as

$$
\widetilde{i}_{\mathrm{F}}=\left(\frac{\partial \bar{i}_{\mathrm{F}}}{\partial V}\right)_{\bar{\gamma}} \widetilde{V}+\left(\frac{\partial \bar{i}_{\mathrm{F}}}{\partial \gamma}\right)_{\bar{V}} \widetilde{\gamma}
$$

or

$$
\widetilde{i}_{\mathrm{F}}=\left(\frac{1}{R_{\mathrm{t}}}\right) \widetilde{V}+\left[K_{\mathrm{X}} \exp \left[b_{\mathrm{X}} \bar{V}\right]-K_{\mathrm{M}} \exp \left[b_{\mathrm{M}} \bar{V}\right]\right] \widetilde{\gamma}
$$

Following

$$
\Gamma \frac{\mathrm{d} \gamma}{\mathrm{d} t}=\frac{i_{\mathrm{M}}}{\mathrm{F}}-\frac{i_{\mathrm{X}}}{\mathrm{F}}
$$

the oscillating component of the surface coverage can be expressed as

$$
\Gamma \mathrm{Fj} \omega \widetilde{\gamma}=\left(\frac{1}{R_{\mathrm{t}, \mathrm{M}}}-\frac{1}{R_{\mathrm{t}, \mathrm{X}}}\right) \tilde{V}-\left(K_{\mathrm{X}} \exp \left(b_{\mathrm{X}} \bar{V}\right)+K_{\mathrm{M}} \exp \left(b_{\mathrm{M}} \bar{V}\right)\right) \widetilde{\gamma}
$$


yielding

$$
\widetilde{\gamma}=\left(\frac{\frac{1}{R_{\mathrm{t}, \mathrm{M}}}-\frac{1}{R_{\mathrm{t}, \mathrm{X}}}}{\Gamma \mathrm{Fj} \omega+\left(K_{\mathrm{X}} \exp \left(b_{\mathrm{X}} \bar{V}\right)+K_{\mathrm{M}} \exp \left(b_{\mathrm{M}} \bar{V}\right)\right)}\right) \widetilde{V}
$$

The faradaic admittance may be derived by taking the ratio of the oscillating faradaic current and the electrode potential, i.e.,

$$
Y_{\mathrm{F}}=\frac{1}{R_{\mathrm{t}}}+\frac{\left(R_{\mathrm{t}, \mathrm{M}}^{-1}-R_{\mathrm{t}, \mathrm{X}}^{-1}\right)\left(K_{\mathrm{X}} \exp \left[b_{\mathrm{X}} \bar{V}\right]-K_{\mathrm{M}} \exp \left[b_{\mathrm{M}} \bar{V}\right]\right)}{\Gamma F \mathrm{j} \omega+K_{\mathrm{X}} \exp \left[b_{\mathrm{X}} \bar{V}\right]+K_{\mathrm{M}} \exp \left[b_{\mathrm{M}} \bar{V}\right]}
$$

Equation (20) may be expressed as a faradaic impedance as

$$
Z_{\mathrm{F}}=\left(\frac{1}{R_{\mathrm{t}}}+\frac{A}{\mathrm{j} \omega+B}\right)^{-1}
$$

in which variables $A$ and $B$ are given by

$$
A=\frac{\left(R_{\mathrm{t}, \mathrm{M}}^{-1}-R_{\mathrm{t}, \mathrm{X}}^{-1}\right)\left(K_{\mathrm{X}} \exp \left[b_{\mathrm{X}} \bar{V}\right]-K_{\mathrm{M}} \exp \left[b_{\mathrm{M}} \bar{V}\right]\right)}{\Gamma \mathrm{F}}
$$

and

$$
B=\frac{K_{\mathrm{X}} \exp \left[b_{\mathrm{X}} \bar{V}\right]+K_{\mathrm{M}} \exp \left[b_{\mathrm{M}} \bar{V}\right]}{\Gamma \mathrm{F}}
$$

respectively.

The impedance of a recessed disk electrode for this system includes an ohmic resistance in series with an interfacial impedance comprising a double layer capacitance and a faradaic impedance in parallel, as shown in Figure 1. The faradaic impedance for a two-step reaction mechanism coupled by an adsorbed intermediate consist of a charge-transfer resistance in parallel to an adsorption impedance $Z_{\text {ads }}$. Equation (21) appears as the parallel combination of $R_{\mathrm{t}}$ and an adsorption impedance expressed as

$$
Z_{\text {ads }}=\frac{B+\mathrm{j} \omega}{A}
$$

Equation (24) is a mathematical expression that cannot be expressed as an equivalent electrical circuit with defined passive elements when $A<0$. 


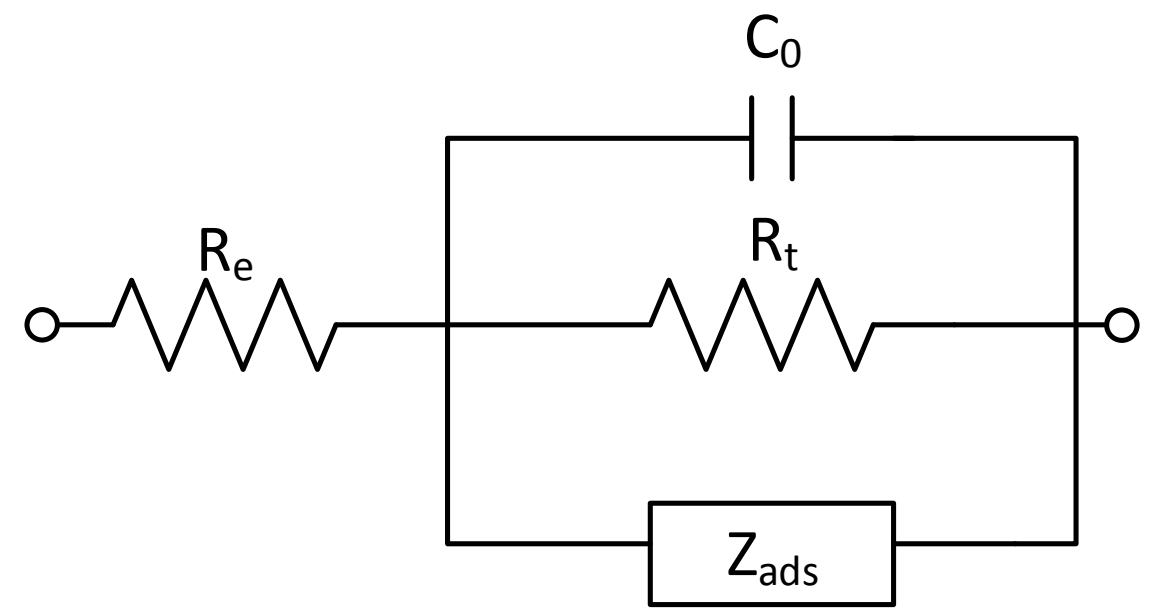

Figure 1: Equivalent circuit diagram for a recessed electrode with reactions coupled by an adsorbed intermediate. The impedance $Z_{\text {ads }}$ is obtained from equation (24).

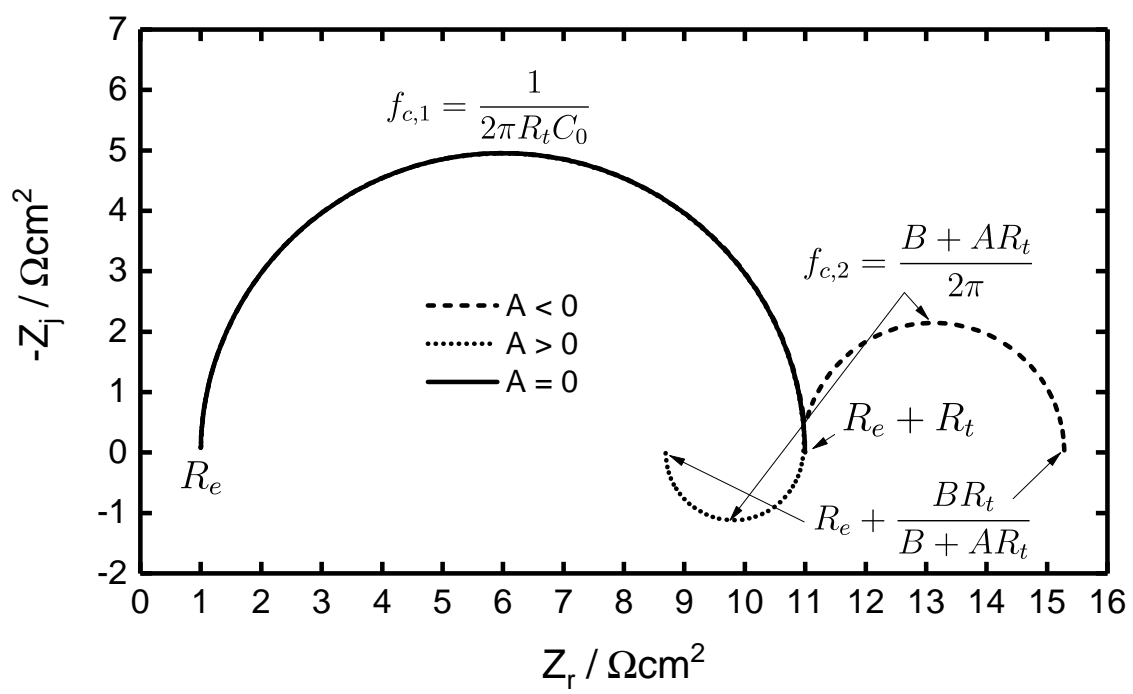

Figure 2: Calculated impedance based on Figure 1 with the faradaic impedance calculated from equation (21) for cases where $A=0, A<0$, and when $A>0$. When $A=0$, the faradaic impedance reduces to $Z_{\mathrm{F}}=R_{\mathrm{t}}$ and the interfacial impedance only exhibits one time constant. When $A<0$, the interfacial impedance shows two capacitive semicircles, and, when $A>0$, the interfacial impedance has a high-frequency capacitive loop and low-frequency inductive loop such that the low-frequency limit for the capacitive loop is $R_{\mathrm{e}}+R_{\mathrm{t}}$. 
The calculated impedance for a recessed electrode is shown in Figure 2 for cases where $A=0, A<0$, and when $A>0$. When $A=0$, the faradaic impedance reduces to $Z_{\mathrm{F}}=R_{\mathrm{t}}$ and the interfacial impedance only exhibits one time constant. When $A<0$, the interfacial impedance shows two capacitive semicircles, and, when $A>0$, the interfacial impedance has a high-frequency capacitive loop and low-frequency inductive loop such that the low-frequency limit for the capacitive loop is $R_{\mathrm{e}}+R_{\mathrm{t}}$. The characteristic frequency of the high-frequency loop is based on the $R_{\mathrm{t}} C_{0}$ time constant. The characteristic frequency of the low-frequency loop is based on the time constant $\tau=1 /\left(B+A R_{\mathrm{t}}\right)$. The low-frequency limit for the impedance is given by

$$
\lim _{\omega \rightarrow 0} Z(\omega)=R_{\mathrm{e}}+\frac{B R_{\mathrm{t}}}{B+A R_{\mathrm{t}}}
$$

for all values of $A$.

\section{Finite-Element Model}

The impedance was simulated by solving Laplace's equation for the potential distribution in the electrolyte domain, expressed as

$$
\nabla^{2} \Phi=0
$$

Following equation (7), the potential may be expressed as

$$
\Phi=\bar{\Phi}+\operatorname{Re}\{\widetilde{\Phi} \exp (j \omega t)\}
$$

The electrolyte domain comprised a 2-D axisymmetric quarter of a circle domain, shown in Figure 3. The counterelectrode was set as the curved boundary with the condition that $\bar{\Phi}=0$ for the steady-state solution and $\widetilde{\Phi}=0$ for the oscillating condition. The working electrode was centered at $r=0$ and $y=0$. The electrolyte domain was 2000 times larger than the radius of the disk. The boundary condition at the working electrode for the steady-state case was expressed as equation (9). The boundary condition at the working electrode for the oscillating case was expressed as

$$
\widetilde{i}=\left(\mathrm{j} \omega C_{0}+R_{\mathrm{t}, \mathrm{M}}^{-1}+R_{\mathrm{t}, \mathrm{X}}^{-1}\right) \widetilde{V}+K_{\mathrm{X}} \exp \left(b_{\mathrm{X}} \bar{V}\right) \widetilde{\gamma}-K_{\mathrm{M}} \exp \left(b_{\mathrm{M}} \bar{V}\right) \widetilde{\gamma}
$$

where $\widetilde{\gamma}$ is given by equation (19). 


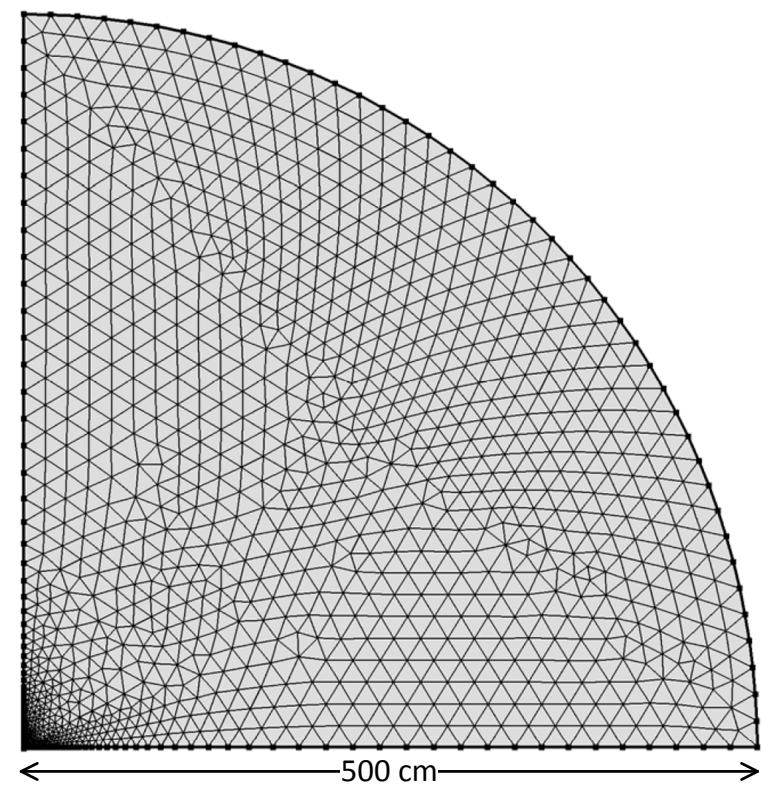

Figure 3: Grid representation of the domain for the finite-element simulations. The disk electrode is located at the origin but is not visible in this scale as the $0.25 \mathrm{~cm}$ disk radius is much smaller than the $500 \mathrm{~cm}$ radius for the calculation domain.

Equations (26), (28), and the boundary condition at the counterelectrode were solved for a range of frequencies, and the global impedance was calculated as the ratio of the applied perturbation $V$ and the total oscillating current at the disk electrode, $\tilde{i}$. The local interfacial impedance may be calculated by probing the local oscillating potential in the electrolyte outside the double layer $\widetilde{\Phi}_{0}(r)$ and may be expressed as

$$
z_{0}(r)=\frac{\widetilde{V}(r)}{\widetilde{i}(r)}
$$

where $\widetilde{V}(r)=\widetilde{\Phi}_{\mathrm{m}}-\widetilde{\Phi}_{0}(r)$. The global interfacial interfacial impedance may be found by integrating the local interfacial impedance across the surface of the whole disk. The global ohmic impedance may be calculated as the difference between the global impedance and the global interfacial impedance as $Z_{\mathrm{e}}=Z-Z_{0}$. 
Table 1: Potential-independent parameters used for the simulations. Lumped potentialdependent parameters $A$ and $B$ may be obtained from equations (22) and (23), respectively. The charge-transfer resistance $R_{\mathrm{t}}$ may be obtained from equations (4), (5), and (11)-(14).

\begin{tabular}{l|l|r|l} 
Symbol & Meaning & Value & units \\
\hline$b_{\mathrm{M}}$ & $\alpha_{\mathrm{M}} \mathrm{F} / \mathrm{RT}$ & 40 & $\mathrm{~V}^{-1}$ \\
$b_{\mathrm{X}}$ & $\alpha_{\mathrm{X}} \mathrm{F} / \mathrm{RT}$ & 10 & $\mathrm{~V}^{-1}$ \\
$K_{\mathrm{M}}$ & Effective reaction constant for Reaction 1 & 77.2 & $\mathrm{~A} / \mathrm{cm}^{2}$ \\
$K_{\mathrm{X}}$ & Effective reaction constant for Reaction 2 & 0.19 & $\mathrm{~A} / \mathrm{cm}^{2}$ \\
$\widetilde{\Phi}_{\mathrm{m}}$ & Perturbation of electrode potential & 10 & $\mathrm{mV}$ \\
$\Gamma$ & Maximum surface coverage of intermediate & $2 \times 10^{-6}$ & $\mathrm{~mol} / \mathrm{cm}^{2}$ \\
$\kappa$ & Electrolyte conductivity & 0.0485 & $\mathrm{~S} / \mathrm{cm}^{2}$ \\
$C_{0}$ & Double-layer capacitance & 20 & $\mu \mathrm{F} / \mathrm{cm}^{2}$
\end{tabular}

\section{Results}

Finite-element simulations were used to simulate the impedance of disk and recessed disk electrodes with reactions coupled by an adsorbed intermediate. The disk geometry produces a radial potential dependence which influences the impedance response. The recessed electrode was used to analyze the effect of heterogenous reaction rates on the impedance response without the confounding effect of the disk geometry. Steady-state results are presented to show the variation of surface properties on the electrodes, and the impedance results are presented to show the effect of the surface distribution. For the sake of brevity, results are only shown for negative values of $A$. Similar frequency dispersion was found for $A>0$.

\subsection{Disk Electrode}

Finite-element simulations were performed to calculate the steady-state potential distribution throughout the electrolyte adjacent to the disk electrode. The potential-independent parameters used in the simulations are presented in Table 1 and were chosen to maximize frequency dispersion due to the disk geometry. Lumped potential-dependent parameters $A$ and $B$ may be obtained from equations (22) and (23), respectively. The charge-transfer resistance $R_{\mathrm{t}}$ may be obtained from equations (4), (5), and (11)-(14). The parameter values presented in Table 1 were selected to conform to the values presented by $\mathrm{Wu}$ et al. [13] and Orazem and Tribollet[20, 21]. These parameters allow observation of three different types of impedance diagrams corresponding to $\langle A\rangle>0,\langle A\rangle=0$, and $\langle A\rangle<0$ at different values of applied 


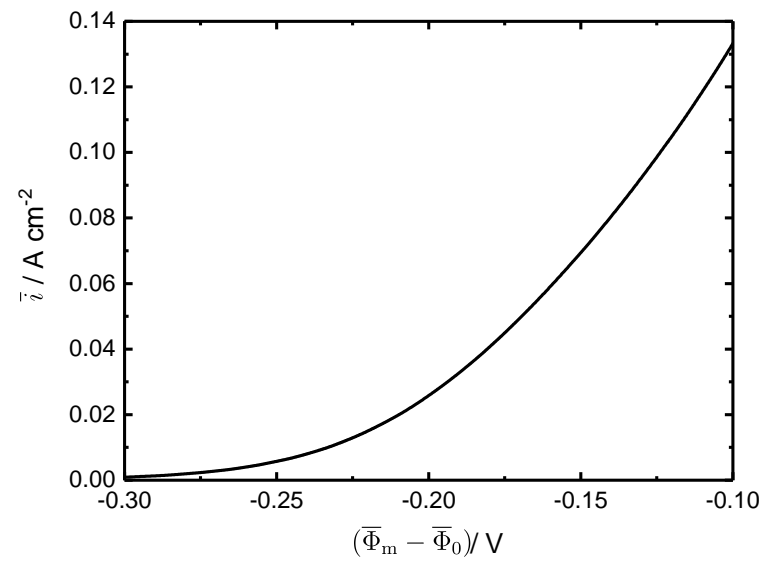

Figure 4: Steady-state current density as a function of surface overpotential for a disk radius $r_{0}=0.25 \mathrm{~cm}$. Simulation parameters are given in Table 1 .

potential. For a temperature of $T=298 \mathrm{~K}$, the values of $\alpha$ corresponding to $b_{\mathrm{M}}=40 \mathrm{~V}^{-1}$ and $b_{\mathrm{X}}=10 \mathrm{~V}^{-1}$ are 1.03 and 0.257 , respectively.

The surface-averaged current density is shown in Figure 4 as a function of the surface overpotential. The current approached zero at more negative potentials. The surface-averaged value of $\langle A\rangle$ is presented in Figure 5 as a function of potential with electrode radius as a parameter. Increases in the disk radius caused the point at which $\langle A\rangle=0$ to shift to more anodic potentials. The steady-state potential outside the double layer for a disk electrode is shown in Figure 6 as a function of radial position. The potential at the edge of the disk is significantly lower than at the center. The parameters associated with the interfacial impedance are potential dependent except for the double-layer capacitance, which was assumed to be independent of potential. Therefore, the interfacial parameters, shown in Figure 7 also vary along the disk surface. Due to the variation of potential, $A$ decreases with increasing radial position and $B$ increases. The charge-transfer resistance also decreases with increasing radial position.

The simulated global impedance $Z$, scaled by the ohmic resistance $R_{\mathrm{e}}=$ $\pi r_{0} / 4 \kappa$, is shown in Figure 8 for an applied potential $\Phi_{\mathrm{m}}=0.037 \mathrm{~V}$ at which $\langle A\rangle=-0.288 \Omega^{-1} \mathrm{~s}^{-1} \mathrm{~cm}^{-2}$. Frequency dispersion is evident as a distortion of both high- and low-frequency loops. The interfacial impedance, shown as $Z_{0}+R_{\mathrm{e}}$, has the appearance of a semicircle for the high-frequency loop and a depressed semicircle for the low-frequency loop. As the interfacial impedance shows low-frequency time-constant dispersion, this effect must be attributed 


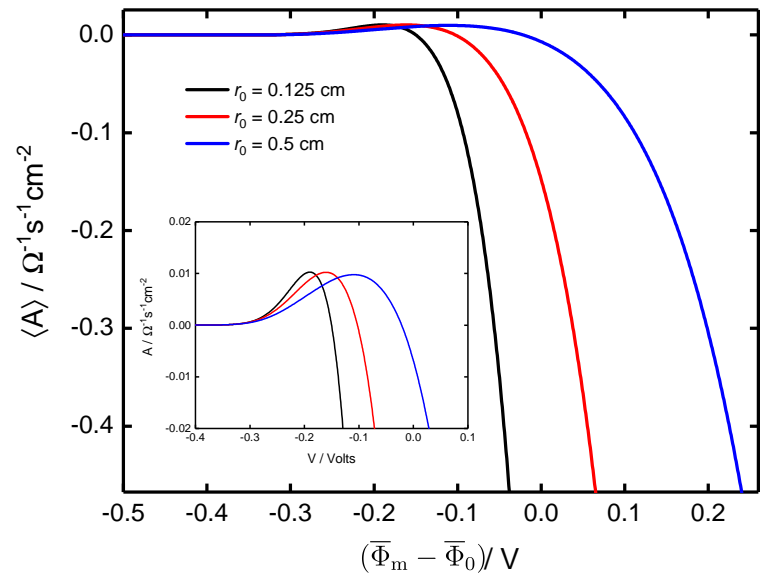

Figure 5: Surface-averaged values for $\langle A\rangle$ as a function of surface overpotential with disk radius as a parameter. Simulation parameters are given in Table 1. The inset shows an expanded view that emphasizes the change of the sign for $\langle A\rangle$.

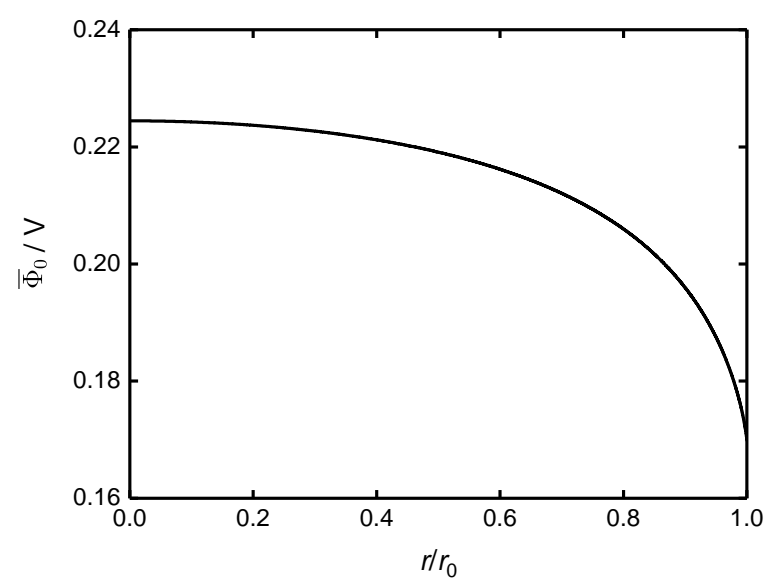

Figure 6: Potential of the electrolyte outside the diffuse double layer as a function of radial position for the simulation presented in Figure 4. 


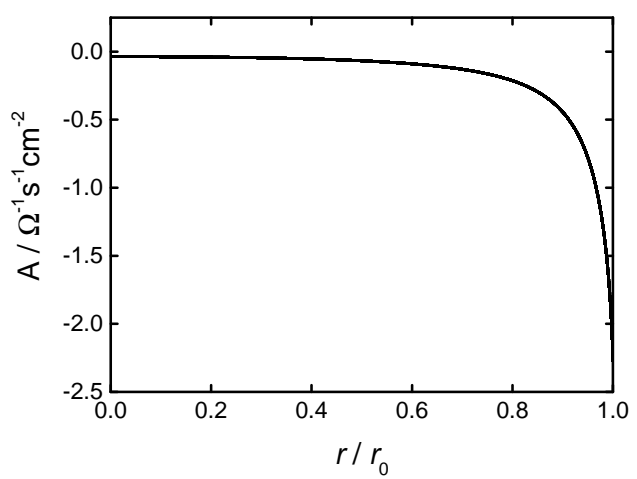

(a)

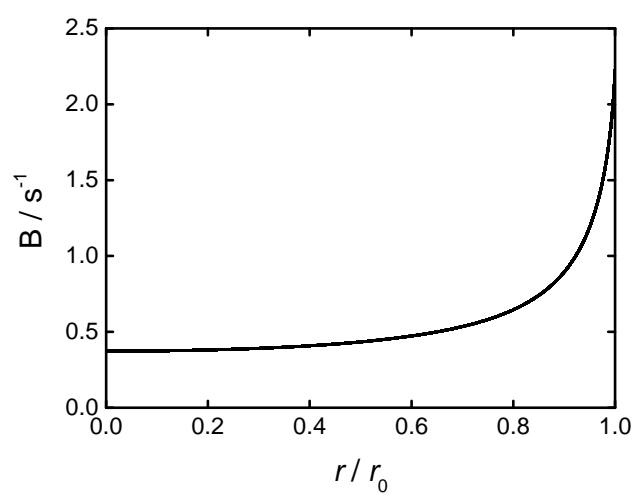

(b)

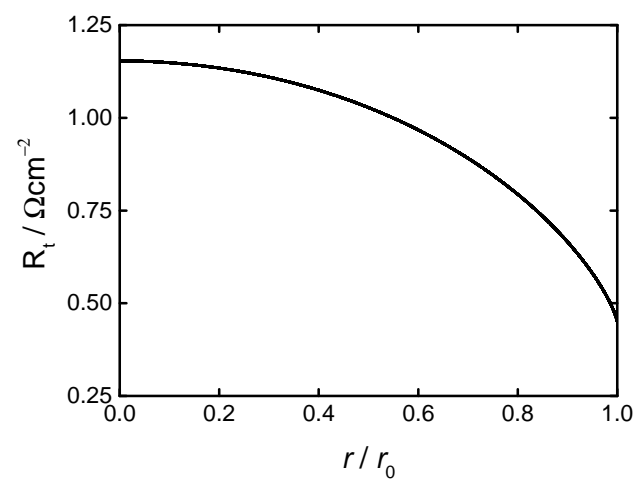

(c)

Figure 7: Steady-state interfacial parameters as a function of dimensionless radial position for the simulation presented in Figure $4 .:$ a) $A$; b) $B$; and c) $R_{\mathrm{t}}$. 


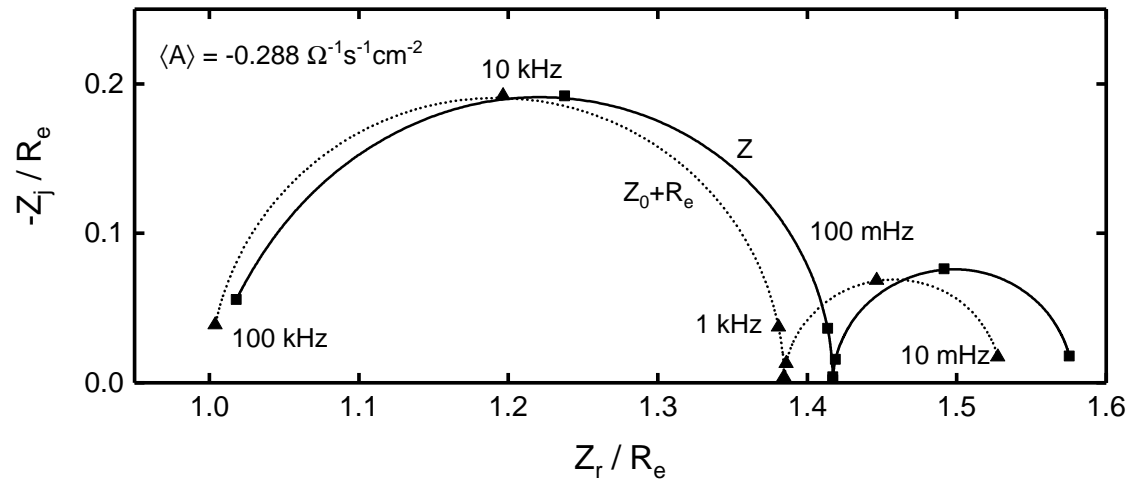

Figure 8: Simulated impedance response in Nyquist format of a disk electrode for an applied potential $\Phi_{\mathrm{m}}=0.037 \mathrm{~V}$ at which $\langle A\rangle=-0.288 \Omega^{-1} \mathrm{~s}^{-1} \mathrm{~cm}^{-2}$. The solid line represents the global impedance response. The dotted line represents the interfacial impedance.

to the faradaic reaction.

The low-frequency dispersion observed for the global impedance includes as well a contribution of the disk geometry. The imaginary part of the global ohmic impedance scaled by the ohmic resistance is shown in Figure 9 as a function of frequency with the radius of the disk as a parameter. The global impedance is represented by the sum of the ohmic and interfacial impedances. A nonzero value for the imaginary part of the ohmic impedance reveals the presence of frequency dispersion. While the magnitude of the imaginary part of the ohmic impedance increases with decreases in the size of the disk and the onset for high-frequency dispersion shifts to higher potentials, the frequency at which low-frequency dispersion appears is comparatively unchanged.

The frequency dispersion associated with the interfacial impedance shown in Figure 8 is caused by the influence of geometry-induced nonuniform potential distributions on the adsorption impedance given as equation (24). The absolute value of the imaginary part of the adsorption impedance, scaled by the ratio $\langle B / A\rangle$, is presented in Figure 10 as a function of the scaled real part of the adsorption impedance with the radius of the disk as a parameter. The imaginary part of the impedance is shown in log-scale to reveal the variation in impedance at low frequencies, shown in the lower-left-hand corner of the figure. The frequency dispersion of the adsorption impedance increases as the disk radius increases. Comparison to simulation results for a recessed electrode shows that the dispersion of the adsorption impedance is caused by geometry-induced nonuniform potential distributions. 


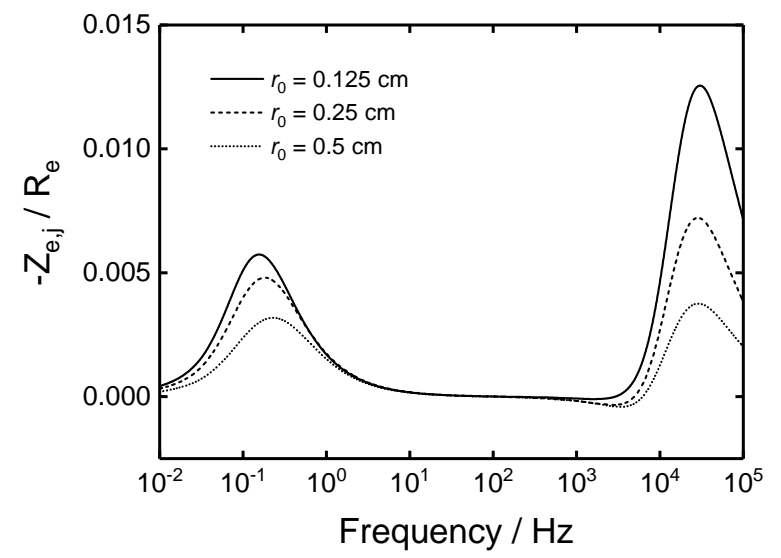

Figure 9: The imaginary part of the ohmic impedance scaled by the ohmic resistance as a function of frequency with disk radius as a parameter for an applied potential $\Phi_{\mathrm{m}}=$ $0.037 \mathrm{~V}$ at which $\langle A\rangle=-0.288 \Omega^{-1} \mathrm{~s}^{-1} \mathrm{~cm}^{-2}$.

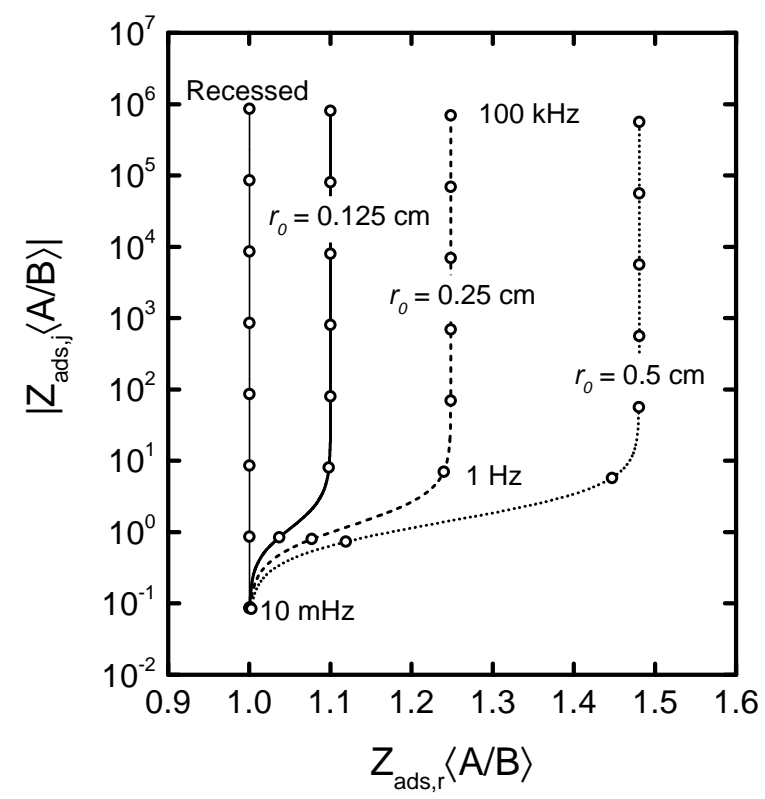

Figure 10: The adsorption impedance scaled by the $\langle B / A\rangle$ with disk radius as a parameter for an applied potential $\Phi_{\mathrm{m}}=0.037 \mathrm{~V}$ at which $\langle A\rangle=-0.288 \Omega^{-1} \mathrm{~s}^{-1} \mathrm{~cm}^{-2}$. The dashed line represents the results for a recessed electrode that eliminates the frequency dispersion caused by disk geometry. 


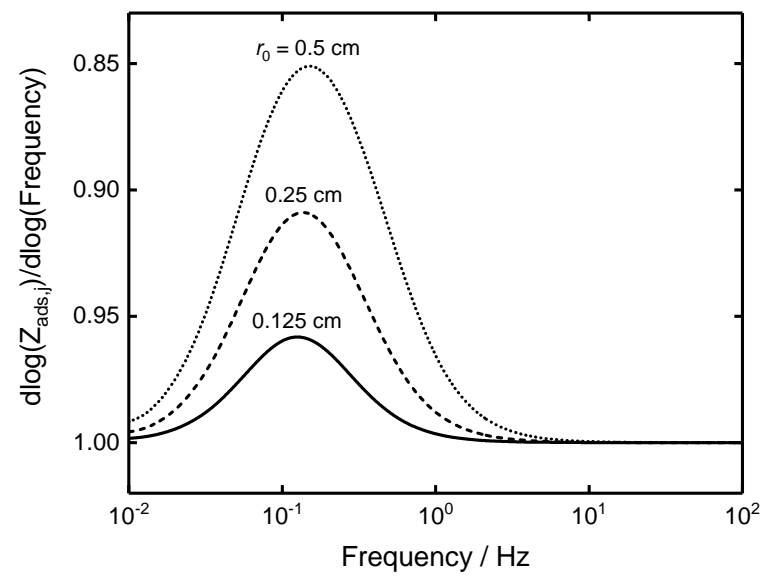

Figure 11: The derivative of the logarithm of the imaginary part of the adsorption impedance with respect to the logarithm of frequency as a function of frequency with the disk radius as a parameter for an applied potential $\Phi_{\mathrm{m}}=0.037 \mathrm{~V}$ at which $\langle A\rangle=-0.288 \Omega^{-1} \mathrm{~s}^{-1} \mathrm{~cm}^{-2}$.

The derivative of the logarithm of the imaginary part of the adsorption impedance with respect to the logarithm of frequency is shown as a function of frequency in Figure 11 with the radius of the disk as a parameter. Frequency dispersion is shown for values which deviate from unity. Therefore, as the radius of the disk electrode increases, the amount of frequency dispersion associated with the interfacial impedance increases. The total amount of frequency dispersion contained within the global impedance is a combination of the interfacial dispersion and the dispersion caused by the ohmic impedance. The ohmic impedance increases as the radius of the disk decreases and the interfacial dispersion decreases as the radius of the disk decreases. The overall frequency dispersion is minimized by using small disk electrodes but unlike the case for a blocking disk electrode, the characteristic frequency associated with the frequency dispersion is not dependent on the size of the disk.

\subsection{Recessed Electrode}

The influence of nonuniform rate constants on impedance response was explored by use of a recessed electrode with a depth to diameter ratio sufficiently large to ensure a uniform current and potential distribution. The rate constants associated with the first and second elementary steps are shown in Figures 12 and 13 as functions of radial position. The rate constants were 


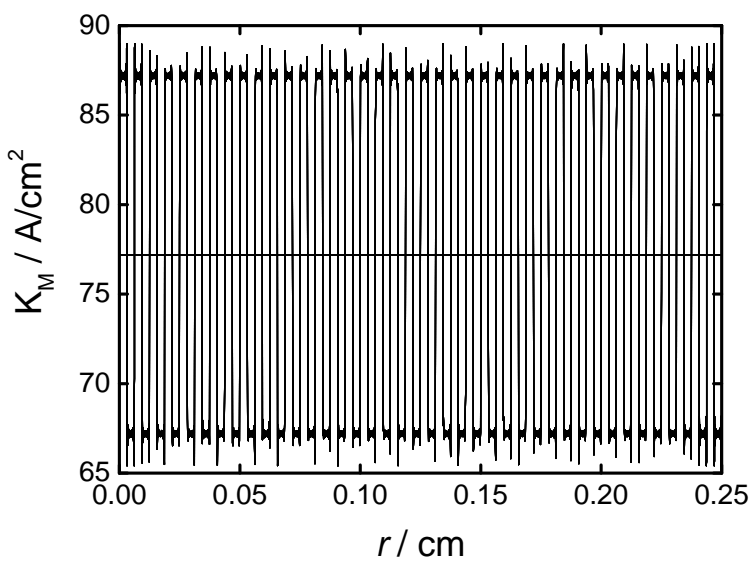

Figure 12: Effective rate constant for Reaction 1 as a function of radial position.

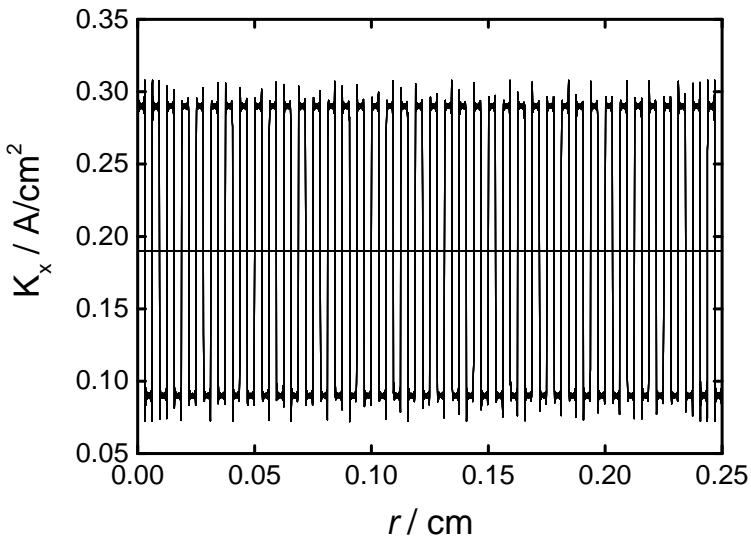

Figure 13: Effective rate constant for Reaction 2 as a function of radial position. 


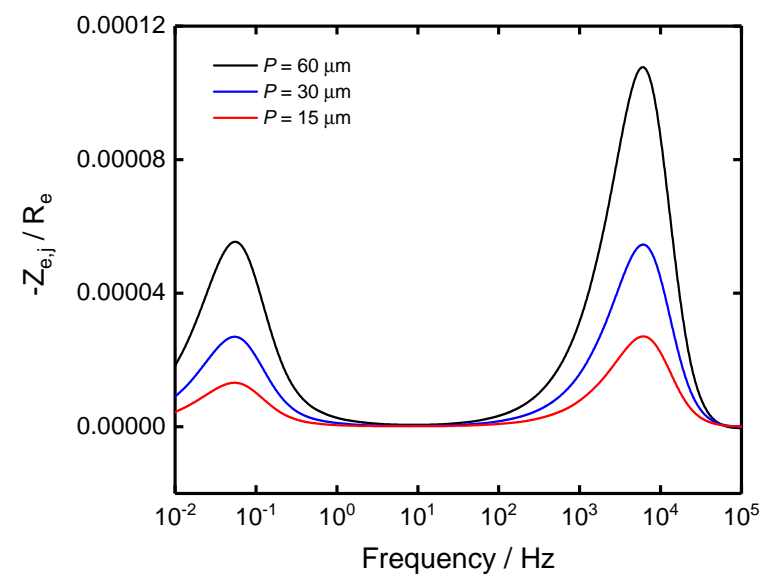

Figure 14: Imaginary part of the ohmic impedance scaled by the ohmic resistance as a function of frequency with the period of the distribution as a parameter. Simulation parameters are given in Table 1 with the exception that rate constants were distributed according to Figures 12 and 13.

varied around a mean value following a square-wave distribution. The square wave form was calculated using a Fourier series with a specified period and amplitude.

A series of calculations were performed to demonstrate that the frequency dispersion associated with the adsorption impedance was not influenced by electrode geometry. The imaginary part of the ohmic impedance scaled by the ohmic resistance is shown in Figure 14 with the period of the heterogeneity as a parameter. The ohmic impedance increased as the period of the distribution increased, but the characteristic frequency associated with the dispersion was unchanged. The derivative of the logarithm of the imaginary part of the adsorption impedance with respect to the the logarithm of frequency is shown in Figure 15 as a function of frequency with the period of the distribution as a parameter. The characteristic frequency associated with the interfacial frequency dispersion did not change with changes in the period of the distribution, and the amount of frequency dispersion increased slightly with decreases in the period of the distribution. Therefore, the frequency at which the dispersion occurs is independent of the period of the rate constant distribution.

To explore the dependence of characteristic frequency on the interfacial parameters, simulations were performed at different potentials, thereby, changing the values of the rate constants. The simulated impedance, scaled 


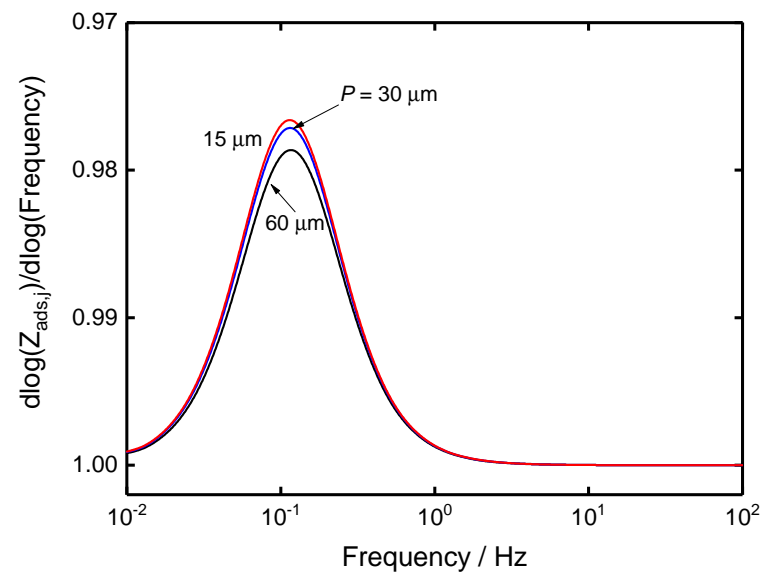

Figure 15: The derivative of the logarithm of the imaginary part of the adsorption impedance with respect to the logarithm of frequency as a function of frequency with the period as a parameter. Simulation parameters are given in Table 1 with the exception that rate constants were distributed according to Figures 12 and 13.

by the surface-averaged charge-transfer resistance, is shown in Figure 16 with steady-state electrode potential as a parameter. Most of the frequency dispersion was confined to the low-frequency capacitive loop. At $\bar{\Phi}_{\mathrm{m}}=0.1 \mathrm{~V}$, an inductive feature is observed that is associated with the variation of $A$ along the surface between positive and negative values. As the potential increased, the frequency dispersion became more significant and the low-frequency loop broadened, reflecting the presence of two distinct time constants. There is minimal frequency dispersion associated with the high-frequency time constant.

The phase angle based on the imaginary part of the impedance [22],

$$
\varphi_{\mathrm{dZj}}=\frac{\mathrm{d} \log \left(Z_{\mathrm{j}}\right)}{\mathrm{d} \log (f)} \times 90^{\circ} .
$$

is presented in Figure 17 as a function of frequency with $\bar{\Phi}_{\mathrm{m}}$ as a parameter. As the potential is shifted in the anodic direction, the frequency dispersion occurs at higher frequencies and the magnitude of the dispersion increases. The same quantity, presented in Figure 18 as a function of the dimensionless frequency,

$$
K=\frac{\omega}{\langle B\rangle+\left\langle A R_{\mathrm{t}}\right\rangle}
$$




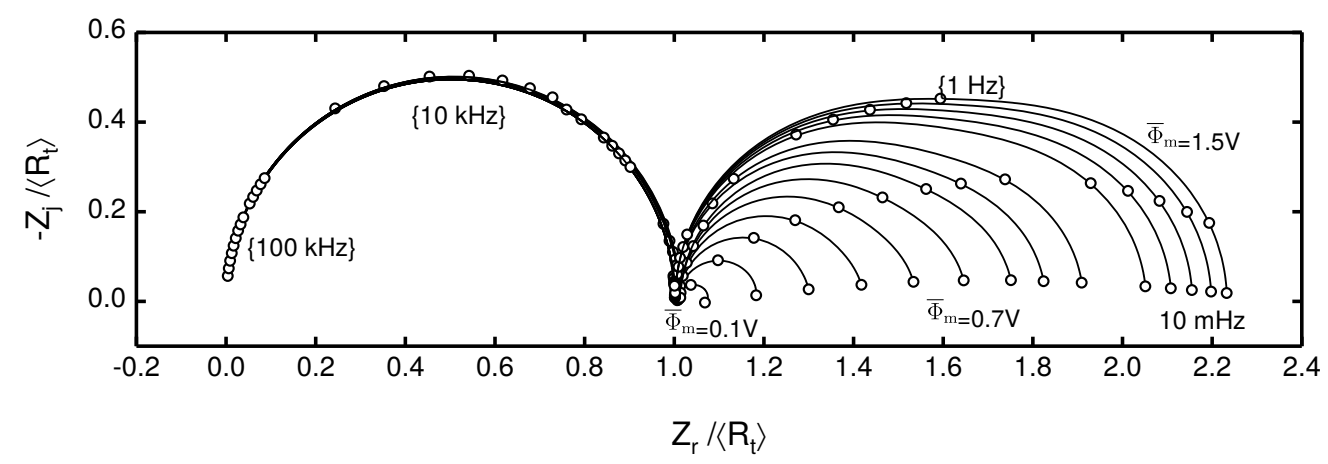

Figure 16: The simulated impedance in Nyquist format scaled by the charge-transfer resistance $\left\langle R_{\mathrm{t}}\right\rangle$ with steady-state electrode potential as a parameter. Simulation parameters are given in Table 1 with the exception that rate constants were distributed according to Figures 12 and 13.

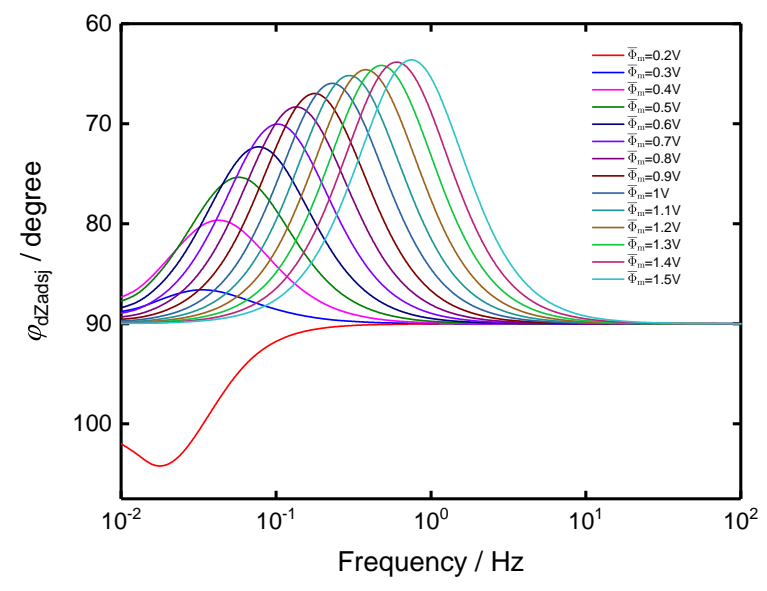

Figure 17: The imaginary-impedance-derived phase angle as a function of frequency with steady-state electrode potential as a parameter. Simulation parameters are given in Table 1 with the exception that rate constants were distributed according to Figures 12 and 13. 


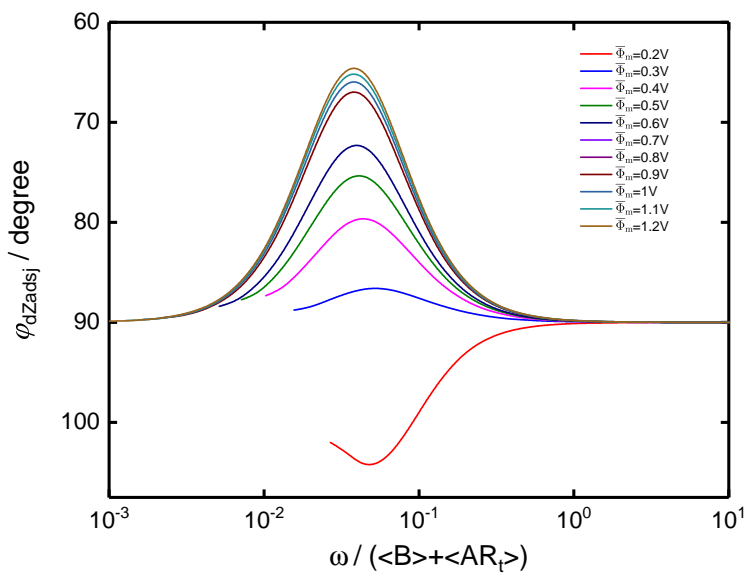

Figure 18: The imaginary-impedance-derived phase angle as a function of dimensionless frequency with steady-state electrode potential as a parameter. Simulation parameters are given in Table 1 with the exception that rate constants were distributed according to Figures 12 and 13.

shows that the low-frequency dispersion occurs at a common value of $K$ as defined by equation (31).

\section{Discussion}

The frequency dispersion observed in the present work arises from the influence of Laplaces equation for potential on the electrochemical reactions coupled by an adsorbed intermediate. For the disk electrode with uniform rate constants, the calculated impedance response for the system shows the influence of geometry-induced current and potential distributions at both high and low frequencies. The resulting frequency dispersion may be described as being caused by the complex frequency-dependent ohmic impedance associated with the disk geometry. No frequency dispersion was seen for a recessed electrode with uniform rate constants because the recessedelectrode geometry yields uniform current and potential distributions. For the recessed electrode with distributed rate constants, the solution of Laplaces equation reveals a local variation of current and potential, resulting in frequency dispersion at low frequency.

Surface rate-constant distributions did not induce frequency dispersion for systems with a single-step reaction.[12] Frequency dispersion was observed at low frequencies for distributions of rate constants for reactions coupled by 


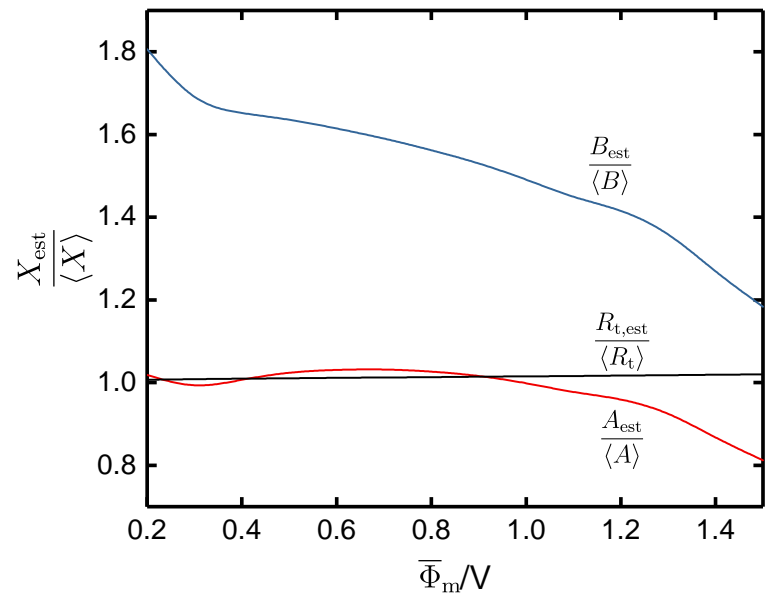

Figure 19: Estimated parameters for the faradaic impedance given as equation (21) obtained by regression of a measurement model to the calculated impedance as a function of steady-state electrode potential. Values are scaled by the corresponding surface-averaged parameters.

an adsorbed intermediate. This dispersion is attributed to the properties of the adsorption impedance given as equation (24).

An analytic study was performed to explore the extent to which frequency dispersion caused by surface distributions of rate constants influences the ability to extract physical properties for systems with adsorbed intermediates. Characteristic frequencies and the high- and low-frequency limits were determined by use of a measurement model[23, 24] in which a series of Voigt elements was used to fit the calculated impedance. The characteristic frequency of the high-frequency time constant was used to calculate $R_{\mathrm{t} \text {,est }}$. The adsorption parameters $A_{\text {est }}$ and $B_{\text {est }}$ were calculated from the low-frequency limit of the impedance and the characteristic frequency of the low-frequency loop.

The resulting estimated parameters, scaled by the respective surfaceaveraged value, are presented in Figure 19 as a function of steady-state potential. The regressed charge-transfer resistance provided a good estimate for the surface-averaged value for all of the potentials simulated. At low potentials, the value of $A_{\text {est }}$ extracted from the simulations agreed with the surface-averaged value; however, at larger potentials, $A_{\text {est }}$ was almost 20 percent smaller than the surface-averaged value. The value of $B_{\text {est }}$ from the simulated impedance could not be reliably determined from the impedance. 
The error in estimation of $B$ was on the order of 20-80 percent from the surface-averaged value. Therefore, for the most reliable interpretation, the charge-transfer resistance may be determined from the high-frequency time constant. However, the adsorption impedance parameters $A$ and $B$ should be estimated with caution from the low frequency time constant. It is advantageous to study systems which comprise adsorbed intermediates with smaller overpotentials as the parameters $R_{\mathrm{t}}$ and $A$ may be estimated reliably.

\section{Conclusions}

On a recessed disk electrode, a distribution of rate constants for reactions coupled by an adsorbed intermediate can give rise to frequency dispersion at both high and low frequencies. For a disk geometry, the geometry-induced nonuniform current and potential distributions make an additional contribution to the complex ohmic impedance. Such frequency dispersions may take the form of a CPE given the right distribution of rate constants, but this CPE behavior would appear over relatively narrow frequency ranges. Thus, a surface distribution of reactions coupled by adsorbed intermediates may provide an explanation for low-frequency CPE behavior, but this will not provide an explanation for the apparent utility of the Brug formula[8] over a broad frequency range.

Simulations of the impedance of a disk electrode with reactions coupled by an adsorbed intermediate showed that frequency dispersion occurs, not

only because of an ohmic impedance caused by the disk geometry, but also because of the potential dependence of the faradaic impedance. Unlike the frequency dispersion associated with a blocking disk electrode, the characteristic frequency of the low-frequency dispersion is independent of the radius of the disk. Nevertheless, use of small disk electrodes will increase the frequency at which high-frequency dispersion is seen and will reduce the amount of low-frequency dispersion.

Simulations performed on recessed electrodes with a periodic square-wave distribution of rate constants showed that the characteristic frequency of the dispersion is dependent on the parameters of the faradaic impedance. In general, the frequency dispersion associated with a distribution of rate constants for reactions coupled by an adsorbed intermediate becomes significant at potentials away from the equilibrium potential. Precaution must be taken in extracting relevant parameters from impedance measurements taken at large overpotentials. 


\section{Acknowledgements}

M. E. Orazem expresses appreciation for financial support associated with the ExxonMobil Chemical Engineering Alumni and University of Florida Research Foundation term professorships. 


\section{References}

[1] J.-B. Jorcin, H. Krawiec, N. Pébère, V. Vignal, Comparison of local electrochemical impedance measurements derived from bi-electrode and microcapillary techniques, Electrochimica Acta 54 (2009) 5775 - 5781.

[2] B. Hirschorn, M. E. Orazem, B. Tribollet, V. Vivier, I. Frateur, M. Musiani, Constant-phase-element behavior caused by resistivity distributions in films: 1. Theory, Journal of the Electrochemical Society 157 (2010) C452-C457.

[3] B. Hirschorn, M. E. Orazem, B. Tribollet, V. Vivier, I. Frateur, M. Musiani, Constant-phase-element behavior caused by resistivity distributions in films: 2. Applications, Journal of the Electrochemical Society 157 (2010) C458-C463.

[4] M. E. Orazem, B. Tribollet, V. Vivier, S. Marcelin, N. Pébère, A. L. Bunge, E. A. White, D. P. Riemer, I. Frateur, M. Musiani, Dielectric properties of materials showing constant-phase-element (CPE) impedance response, Journal of the Electrochemical Society 160 (2013) C215-C225.

[5] E. A. White, M. E. Orazem, A. L. Bunge, Characterization of damaged skin by impedance spectroscopy: Chemical damage by dimethyl sulfoxide, Pharmaceutical Research 30 (2013) 2607-2624.

[6] M. Musiani, M. E. Orazem, N. Pébère, B. Tribollet, V. Vivier, Determination of resistivity profiles in anti-corrosion coatings from constantphase-element parameters, Progress in Organic Coatings 77 (2014) 2076-2083.

[7] A. S. Nguyen, M. Musiani, M. E. Orazem, N. Pébère, B. Tribollet, $\mathrm{V}$. Vivier, Impedance analysis of the distributed resistivity of coatings in dry and wet conditions, Electrochimica Acta 179 (2015) 452-459.

[8] G. J. Brug, A. L. G. van den Eeden, M. Sluyters-Rehbach, J. H. Sluyters, The analysis of electrode impedances complicated by the presence of a constant phase element, Journal of Electroanalytical Chemistry 176 (1984) 275-295. 
[9] P. Córdoba-Torres, T. J. Mesquita, O. Devos, B. Tribollet, V. Roche, R. P. Nogueira, On the intrinsic coupling between constant-phase element parameters $\alpha$ and $q$ in electrochemical impedance spectroscopy, Electrochimica Acta 72 (2012) 172-178.

[10] P. Córdoba-Torres, T. J. Mesquita, R. P. Nogueira, Relationship between the origin of constant-phase element behavior in electrochemical impedance spectroscopy and electrode surface structure, The Journal of Physical Chemistry C 119 (2015) 4136-4147.

[11] C. L. Alexander, B. Tribollet, M. E. Orazem, Contribution of surface distributions to constant-phase-element ( $\mathrm{CPE}$ ) behavior: 2. capacitance, Electrochimica Acta 188 (2016) 566-573.

[12] C. L. Alexander, B. Tribollet, M. E. Orazem, Influence of micrometricscale electrode heterogeneity on electrochemical impedance spectroscopy, Electrochimica Acta 201 (2016) 374-379.

[13] S.-L. Wu, M. E. Orazem, B. Tribollet, V. Vivier, Impedance of a disk electrode with reactions involving an adsorbed intermediate: Local and global analysis, Journal of the Electrochemical Society 156 (2009) C28C38.

[14] S.-L. Wu, M. E. Orazem, B. Tribollet, V. Vivier, Impedance of a disk electrode with reactions involving an adsorbed intermediate: Experimental and simulation analysis, Journal of the Electrochemical Society 156 (2009) C214-C221.

[15] R. D. Armstrong, R. E. Firman, H. R. Thirsk, Ac impedance of complex electrochemical reactions, Faraday Discussions 56 (1973) 244-263.

[16] I. Epelboin, M. Keddam, Faradaic impedances: Diffusion impedance and reaction impedance, Journal of the Electrochemical Society 117 (1970) 1052-1056.

[17] L. Péter, J. Arai, H. Akahoshi, Impedance of a reaction involving two adsorbed intermediates: Aluminum dissolution in non-aqueous lithium imide solutions, Journal of Electroanalytical Chemistry 482 (2000) 125 $-138$. 
[18] S. K. Roy, M. E. Orazem, B. Tribollet, Interpretation of low-frequency inductive loops in pem fuel cells, Journal of the Electrochemical Society 154 (2007) B1378-B1388.

[19] M. Mathias, D. Baker, J. Zhang, Y. Liu, W. Gu, Frontiers in application of impedance diagnostics to h2-fed polymer electrolyte fuel cells, ECS Transactions 13 (2008) 129-152.

[20] M. E. Orazem, B. Tribollet, Electrochemical Impedance Spectroscopy, John Wiley \& Sons, Hoboken, 2008.

[21] M. E. Orazem, B. Tribollet, Electrochemical Impedance Spectroscopy, John Wiley \& Sons, Hoboken, 2 edition, 2017.

[22] C. L. Alexander, B. Tribollet, M. E. Orazem, Contribution of surface distributions to constant-phase-element (CPE) behavior: 1. influence of roughness, Electrochimica Acta 173 (2015) 416-424.

[23] P. Agarwal, M. E. Orazem, L. H. García-Rubio, Measurement models for electrochemical impedance spectroscopy: 1. Demonstration of applicability, Journal of the Electrochemical Society 139 (1992) 1917-1927.

[24] M. E. Orazem, P. T. Wojcik, M. Durbha, I. Frateur, L. H. García-Rubio, Application of measurement models for interpretation of impedance spectra for corrosion, Materials Science Forum 289-292 (1998) 813-828. 